\title{
Analysis of Tumor Vascularization By- Microvessel Density and Cd 34 by Immunohistochemistry- It's Prognostic Significance in Differentiating Benign and Malignant Ovarian Surface Epithelial Tumors
}

\author{
Dr. Shyamala Srujana ${ }^{1 *}$, Dr. SSS Quadri ${ }^{1}$, Dr. N. Srimani ${ }^{2}$ \\ ${ }^{1}$ Associate Professor, Department of Pathology, Government Medical College, Mahabubnagar, Telangana, India \\ ${ }^{2}$ Professor, Department of Pathology, Gandhi Medical College, Secunderabad, Telangana 500003, India
}

DOI: $10.36348 /$ sjpm.2019.v04i11.001

| Received: 12.10 .2019 | Accepted: 24.10.2019| Published: 06.11.2019

*Corresponding author: Dr. Shyamala Srujana

\section{Abstract}

Ovarian neoplasms have become increasingly important not only because of the large variety of neoplastic entities but more so because they have gradually increased mortality due to female genital cancers. Ovarian carcinoma represents the sixth most common female cancers and the fourth leading cause of death due to cancers in women. Surface epithelial tumors were commonest variety of ovarian tumors followed by germ cell tumors. Angiogenesis is a critical factor in tumor growth and metastasis, because tumor proliferation is severely limited by nutrient supply to proliferating tumor cells. Hence tumorigenesis of malignant neoplasms associated with extensive neovascularization. The analysis of tumor vascularization by microvessel density (MVD) and it's prognostic significance has been evaluated in many tumors including ovary. Many studies have established MVD as an important prognostic factor in solid tumors, but there is paucity of literature regarding characteristics of the tumor blood vessels in ovarian surface epithelial tumors. The present study aims to evaluate not just the density of microvessels in ovarian surface epithelial tumors but also the maturity characteristics of proliferating tumor blood vessels with the aid of IMMUNOHISTOCHEMISTRY there by differentiating between benign and malignant epithelial tumors of ovary and predicting the prognosis. Objectives: 1) To determine the incidence of ovarian surface epithelial tumors at our hospital. 2) To clarify the differences in angiogenesis between benign and malignant epithelial tumors. Study Design: Cross sectional study for a period of 15 months (From May 2017 to July 2018) Conducted at Department of Pathology, Gandhi Hospital/Gandhi Medical College, Secunderabad. Materials and Methods: Specimens of ovarian tumors received from Departments of Surgery and OBG Gandhi Hospital/Gandhi Medical College, Secunderabad and Departments of OBG, Government maternity Hospital, Petlaburg, Hyderabad were included in the study. After detailed gross examination of the specimens sections were given for histopathological examination.Immunohistochemistry is performed using standard protocol methods. The monoclonal antibodies used are ANTI CD-34 for endothelial cells in the tumor vessels. Observations and Results: Incidence of ovarian surface epithelial tumours at our hospital is $48.37 \%$. Serous tumours are most common tumours $(60 \%)$ followed by mucinous tumours (40\%). Malignant ovarian tumours showed higher production of immature blood vessels compared to benign tumours. Therefore Mean Micro Vessel Density is higher in malignant tumours as compare to benign tumours. In view of these results, new therapy should aim to alter ratio of immature to mature blood vessels and also Micro vessel density of malignant tumours for a successful outcome.

Keywords: Tumor Vascularization, Microvessel Density, Immunohistochemistry.

Copyright @ 2019: This is an open-access article distributed under the terms of the Creative Commons Attribution license which permits unrestricted use, distribution, and reproduction in any medium for non-commercial use (NonCommercial, or CC-BY-NC) provided the original author and sources are credited.

\section{INTRODUCTION}

Ovarian carcinomas accounts for $3 \%$ of all cancers in females [1]. Ovarian carcinomas represent sixth most common female cancers and fourth leading cause of death due to cancers in women. Surface epithelial tumours are commonest variety of ovarian tumours followed by germ cell tumours [2].
Epithelial ovarian cancers originate from normal ovarian surface epithelium or from inclusion cysts arising from surface epithelium [3]. Recent evidence shows that high grade serous carcinoma arises from fallopian tube [4]. This disease begins in and usually limited to peritoneal cavity [5]. Owing to 
paucity of symptoms and insidious in onset, most patients present with advanced disease and 5 years survival rates are approximately $20 \%$ [6]. It is diagnosed at advanced stage when it has spread to secondary sites already. The common histological types of surface epithelial tumours are serous and mucinous tumours [7]. The surface epithelium consists of single layer of modified mesothelial cells varying from flat to cuboidal to columnar. Stroma consists of spindle shaped cells with scant cytoplasm arranged in storiform pattern. The peripheral zone of stroma is called as cortex which contains numerous follicles in various stages of development.

Surface epithelial tumours are divided into two broad categories: Type I and Type II, based on clinicopathological features and characteristic molecular genetic changes [8].

Type I and type II refer to tumorigenic pathways and are not histopathologic diagnostic terms. Type I tumours are low grade, indolent neoplasms which arise from precursor lesions [atypical proliferative tumours and endometriosis], and present as large stage I neoplasms. This group includes low grade serous (invasive micropapillary serous carcinoma, MPSC) low grade endometrioid, mucinous and tentatively clear cell carcinomas. Clear cell carcinoma has features of type I like its association with endometriosis, but is high grade. Type I tumours harbor somatic mutations of genes-KRAS, BRAF, PIK3CA, ERBB2 and signaling molecules including PTEN and $\beta$-catenin [8]. Type II tumours are high-grade serous carcinomas which are aggressive and high-grade. They have been said to arise "denovo" in the past. Recent data suggests that they arise from intraepithelial carcinomas, detected in tubal fimbriae. More than $75 \%$ of type II carcinomas have TP53 mutations and present as stage III tumours [8].

The epithelial tumours are classified as benign, borderline, and malignant. The benign tumours are often subclassified based on components of tumours, which include cystic areas (cystadenomas), cystic and fibrous areas (cystadenofibromas), and predominantly fibrous areas (adenofibromas). The borderline and malignant tumours can have cystic component [1].

Angiogenesis is critical factor in tumour growth and metastasis, because tumour proliferation is severely limited by the nutrient supply to proliferating tumour cells [5]. Hence tumorigenesis of malignant neoplasms is associated with extensive neovascularisation. The analysis of tumour vascularisation by microvessel density and its prognostic significance has been evaluated in many tumours [8-13]. But there is paucity of literature regarding characteristics of tumour blood vessels in ovarian surface epithelial tumours. CD34 is cell surface protein expressed in endothelial cells. Upregulation of
CD34 is associated with increased neovascularisation during tumour development [9].

\section{AIMS AND OBJECTIVES}

- To determine the incidence of ovarian surface epithelial tumours at our hospital.

- To study the differences in angiogenesis between benign and malignant epithelial tumours using immunohistochemistry

- To assess characteristics of tumour vessels with aid of immunohistochemistry.

\section{MATERIALS AND METHODS}

Specimens of ovarian tumors received from Departments of Surgery and OBG Gandhi Hospital/Gandhi Medical College, Secunderabad and Departments of OBG, Government maternity Hospital, Petlaburg, Hyderabad were included in the study. After detailed gross examination of the specimens sections were given for histopathological examination.Immunohistochemistry is performed using standard protocol methods. The monoclonal antibodies used are ANTI CD-34 for endothelial cells in the tumor vessels.

\section{Inclusion Criteria}

Properly labelled and formalin fixed oophorectomy specimens And Surface epithelial tumours only

\section{Exclusion Criteria}

- Other ovarian tumours

- Unfixed and Autolysed samples.

The specimens were received in formalin. After gross examination, tissues were routinely processed, embedded in paraffin and five-micron thick sections were taken.

Sections were stained with conventional Hematoxylin and Eosin stains by the following procedure.

Micro Vessel Density (MVD) was calculated from CD34 stained sections by using the method proposed by Weidner et al., [15] In each section, three most vascular areas were chosen using low power field $(10 X)$ - HOT SPOTS - Figure-1. Number of micro vessels in each hotspot is counted using high power field (40x) and average of three hotspots is recorded. 


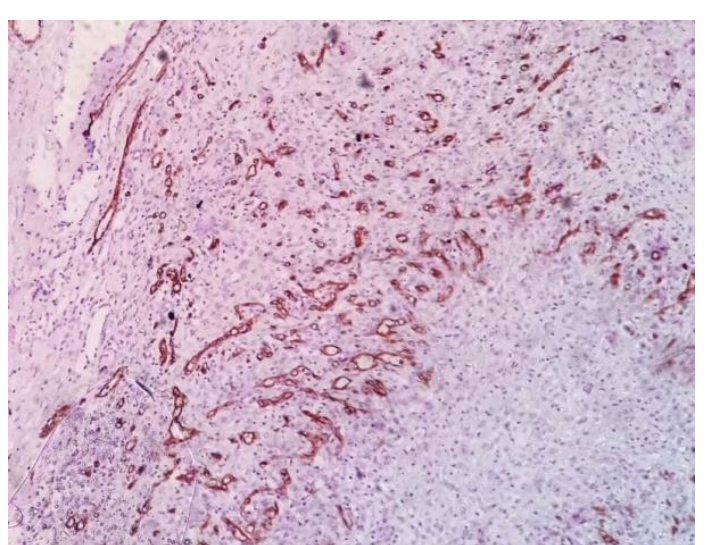

Fig-1: IHC Stain- CD34 - 10X-serous carcinoma

\section{Showing Hotspot}

- Brown staining of endothelial cells, separated from adjacent microvessels, connective tissue and tumour cells is considered as single countable microvessel.

- $\quad$ Single cell / clusters counted

- Vessel lumen is not required for identification of microvessel.

- Large vessels with thick muscular walls and large vessels with lumina greater than approximately $8 \mathrm{RBC}$ 's were excluded from count (Figure-2).

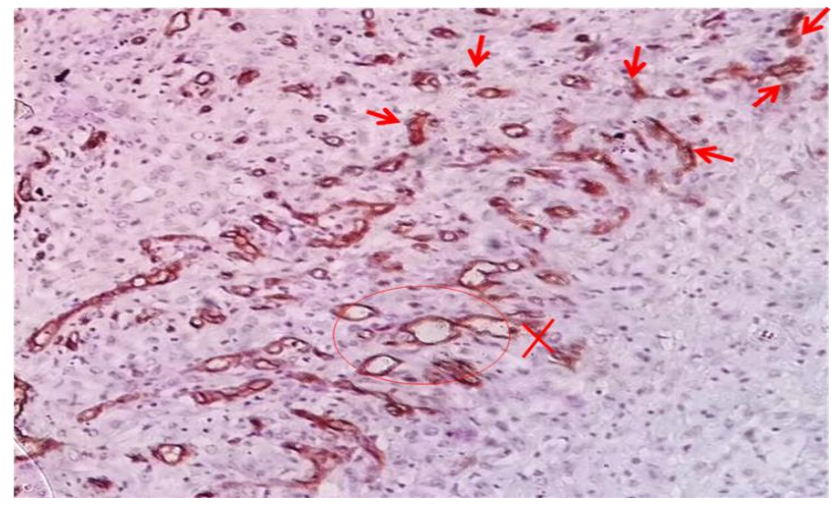

Fig-2: IHC Stain - CD34 - 40X - Serous carcinoma Large lumen vessels are excluded

Few microvessels which can be counted are shown by arrows

- Counts are expressed as total number of micro vessels per hpf 40x $\left(0.196 \mathrm{~mm}^{2}\right)$.

- This data is referred as micro vessel density counts.

- In each section, three most vascular areas were chosen using low power field (10x) - Hot spots.

\section{OBSERVATIONS AND RESULTS}

Youngest age at diagnosis -29 years; oldest -65 years. Most of the patients belong to age group -40 to 49 years - 20 cases_Total number of ovarian tumours received at Department of Pathology, Gandhi hospital in during period a of 15 months study are 70, among which 34 are surface epithelial tumours. 4 cases are excluded from the study due to autolysis. Incidence of ovarian surface epithelial tumours at our hospital is $48.57 \%$.

Among 30 cases of Ovarian Surface epithelial tumours;

- Benign cases - $15(50 \%)$,

- Borderline cases - $3(10 \%)$ and

- Malignant cases - 12(40\%).

Among the tumours,

- Serous tumors- 18 cases $(60 \%)$

- Mucinous tumours -11 cases ( $37 \%$ ) and

- Brenner tumours-1 cases (3\%)

Table-1: Histomorphological Distribution of Surface Epithelial Tumours of Ovary

\begin{tabular}{|l|l|l|l|}
\hline Type of Tumour & $\begin{array}{c}\text { Benign } \\
(\mathbf{5 0 \%})\end{array}$ & $\begin{array}{c}\text { Borderline } \\
(\mathbf{1 0 \%})\end{array}$ & $\begin{array}{l}\text { Malignant } \\
(\mathbf{4 0 \%})\end{array}$ \\
\hline $\begin{array}{l}\text { SEROUS TUMOURS } \\
\mathrm{n}=18(60 \%)\end{array}$ & 10 & 02 & 06 \\
\hline $\begin{array}{l}\text { MUCINOUS TUMOURS } \\
\mathrm{n}=11(37 \%)\end{array}$ & 07 & 01 & 03 \\
\hline $\begin{array}{l}\text { BRENNER TUMOURS } \\
\mathrm{N}=1(3 \%)\end{array}$ & 00 & -- & 01 \\
\hline
\end{tabular}


Table-2: Frequency Distribution of Surface Epithelial Tumours of Ovary

\begin{tabular}{|l|l|}
\hline Histomorphological Type & Number of Cases $(\mathbf{n = 3 0})$ \\
\hline Serous Cystadenoma & $\mathbf{0 8}$ \\
\hline Serous Adenofibroma & 02 \\
\hline Serous Borderline Tumour & 02 \\
\hline Serous Carcinoma & 06 \\
\hline Mucinous Cystadenoma & $\mathbf{0 6}$ \\
\hline Mucinous Borderline Tumour & 01 \\
\hline Mucinous Carcinoma & 04 \\
\hline Malignant Brenner Tumour & 01 \\
\hline
\end{tabular}

NOTE: Serous cystadenomas are most common tumours followed by mucinous cystadenomas.

Table-3: Age Distribution.

\begin{tabular}{|l|l|}
\hline Age & Number of Patients \\
\hline $20-29$ & 01 \\
\hline $30-39$ & 03 \\
\hline $40-49$ & 20 \\
\hline $50-59$ & 05 \\
\hline $60-69$ & 01 \\
\hline Total & 30 Cases \\
\hline
\end{tabular}

NOTE: Majority (20) cases belong to age group of 4049 years.

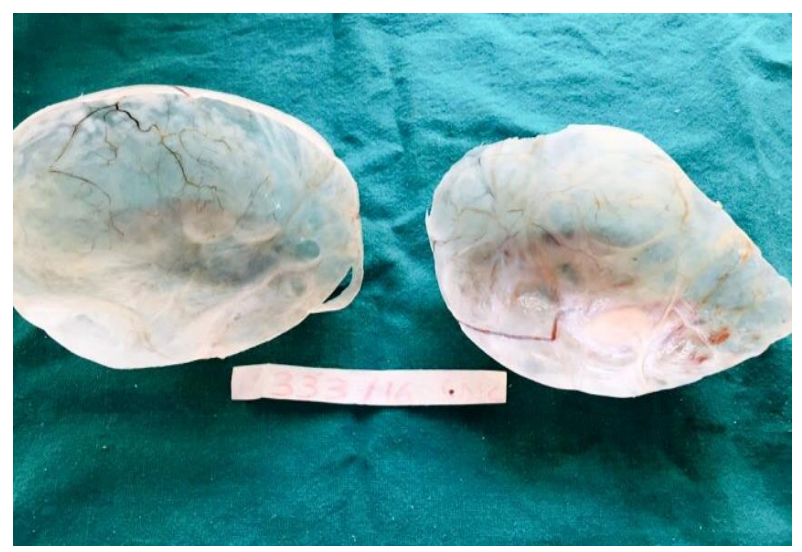

Fig-1: Gross Specimen - Cut surface of Benign Serous Cystadenoma showing unilocular, glistening, smooth cyst wall

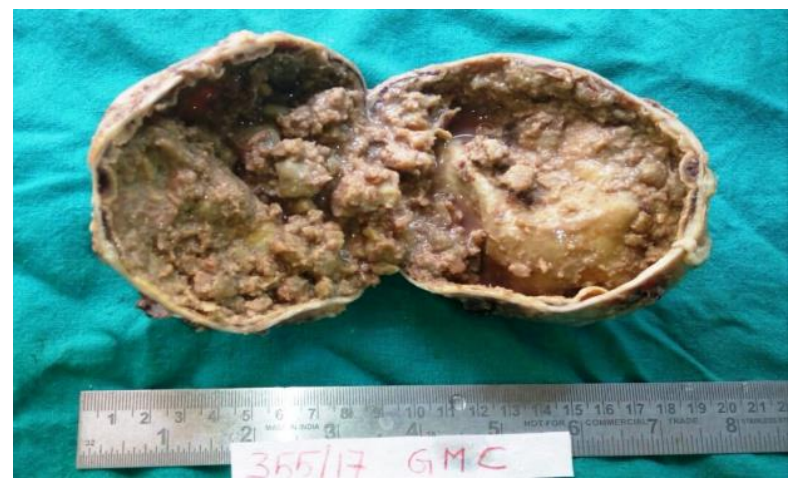

Fig-2: Gross Specimen - cut surface of Papillary Serous Carcinoma showing papillary fronds

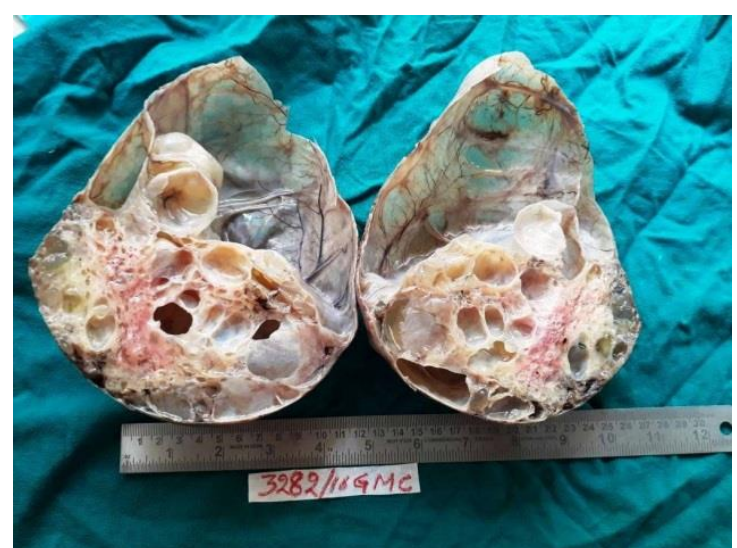

Fig-3: Gross Specimen - Cut surface of Benign Mucinous Cystadenoma showing multilocular cyst wall with mucinous area

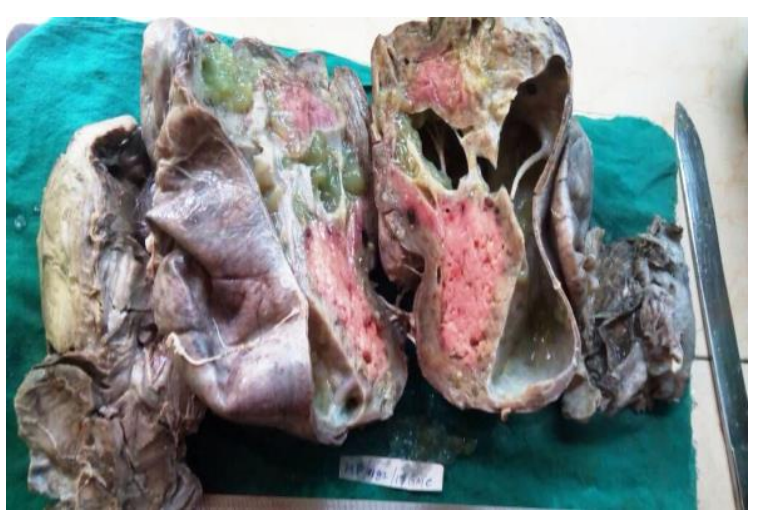

Fig-4: Gross specimen- Cut surface of Mucinous Carcinoma showing solid and mucinous areas

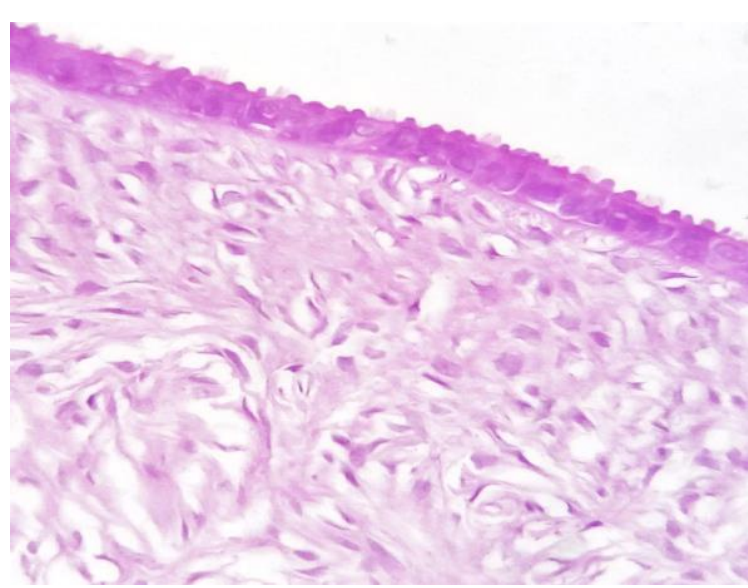

Fig-5: H \& E Stain- 40X - Serous Cystadenoma showing cyst wall lined by cuboidal epithelium 


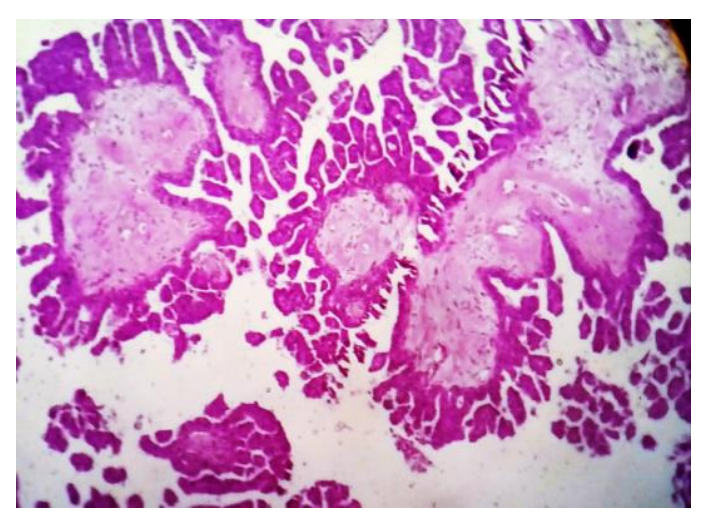

Fig-6: H \& E Stain- 40X - Serous Carcinoma showing papillae with fibrovascular core lined by malignant cells

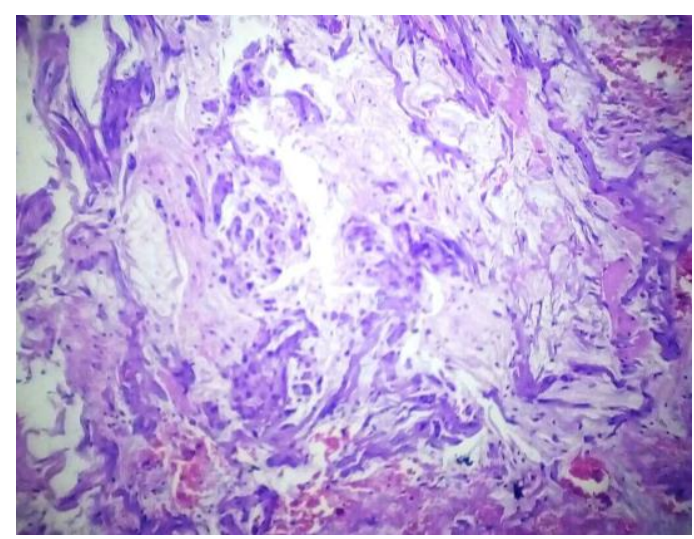

Fig-7: H \& E Stain- 40X - Mucinous Carcinoma showing pools of mucin

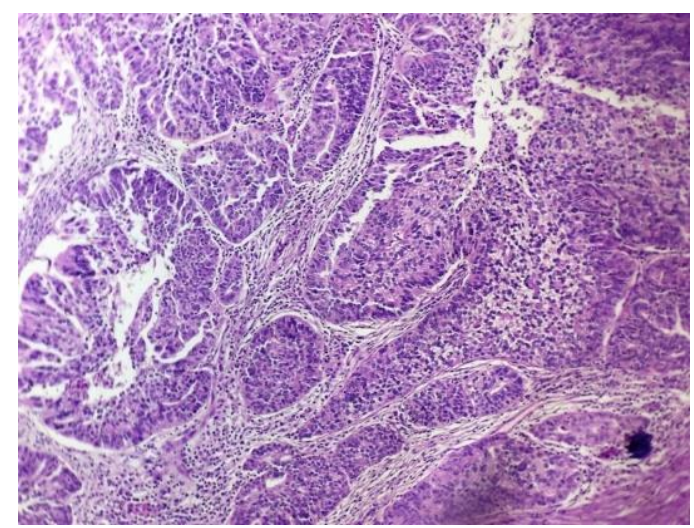

Fig-8: H\&E Stain-40X- malignant Brenner tumour showing malignant transitional epithelium with focus of benign island

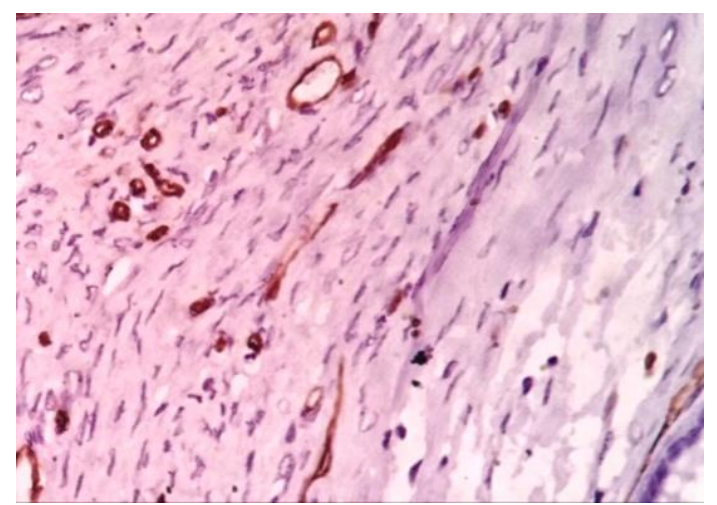

Fig-9: IHC Stain- CD 34- 40X- Benign mucinous cystadenoma showing low microvessel density

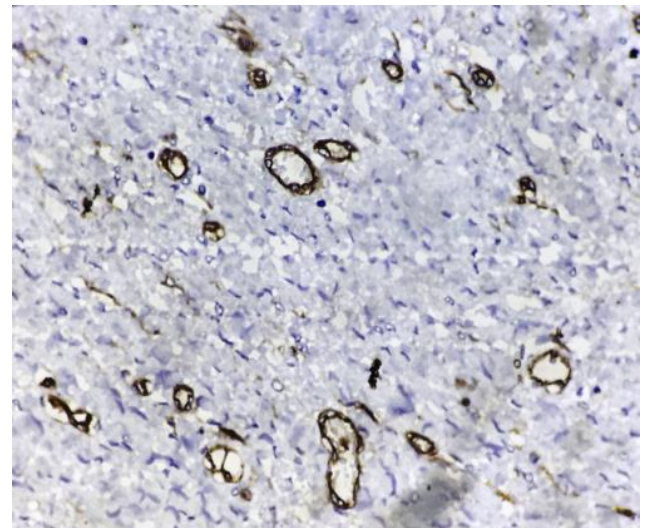

Fig-10: IHC stain - CD 34- 40x- Benign serous cystadenoma showing low microvessel density

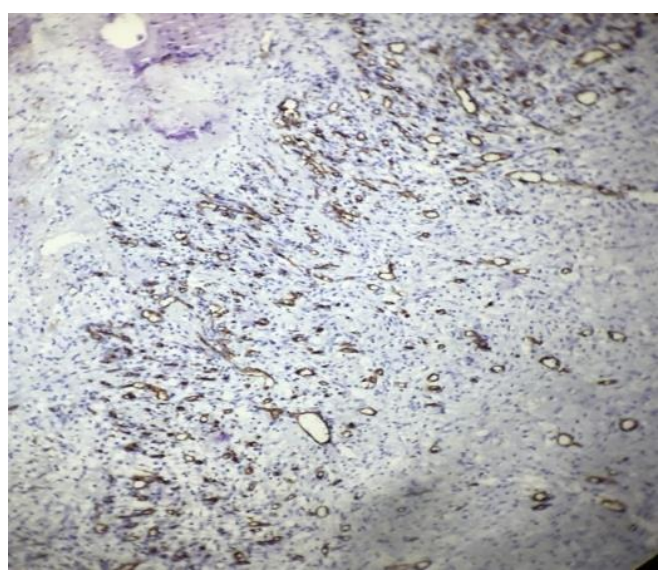

Fig-11: IHC Stain- CD 34 -10X- serous carcinoma showing hot spot

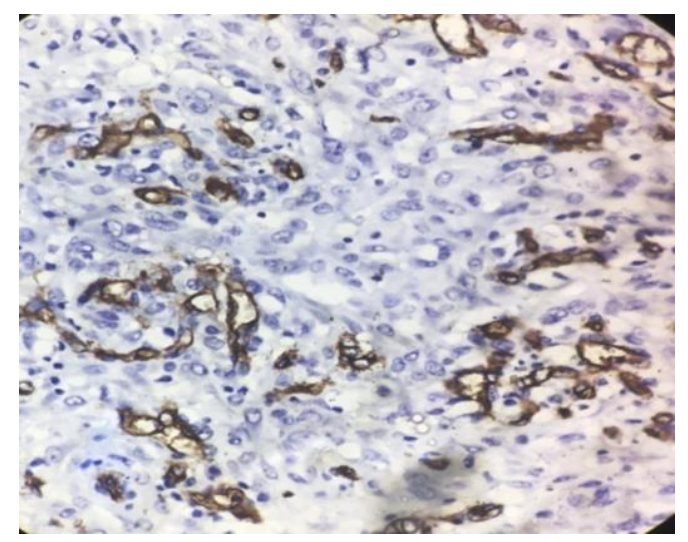

Fig-12: IHC Stain-CD34- 40X- Mucinous carcinoma showing high microvessel density 
Table-4: Micro Vessel Density of Benign ovarian surface epithelial Tumours

\begin{tabular}{|l|l|l|}
\hline Benign Tumours & Number of Cases & Mean MVD / HPF (SD) \\
\hline Serous Cystadenomas & 10 & 4.50 \\
\hline Mucinous Cystadenoma & 05 & 4.33 \\
\hline Total & 15 & $4.48(0.99)$ \\
\hline
\end{tabular}

NOTE: Mean microvessel density per high power field of benign ovarian surface epithelial tumours is $4.48 \pm 0.99$

Table-5: Micro Vessel Density of Borderline ovarian surface epithelial Tumours

\begin{tabular}{|l|l|l|}
\hline Borderline Tumours & Number of Cases & Mean MVD/HPF \\
\hline Serous Borderline Tumour & 02 & 7.5 \\
\hline Mucinous Borderline Tumour & 01 & 7.5 \\
\hline Total & 03 & 7.5 \\
\hline
\end{tabular}

NOTE: Mean micro vessel density per high power field of borderline ovarian surface epithelial tumours is 7.5

As only3 cases of borderline ovarian surface epithelial tumours are included, standard deviation is not calculated

Table-6: Micro Vessel Densityof Malignant ovarian surface epithelial tumours

\begin{tabular}{|l|l|l|}
\hline Malignant Tumours & Number of cases & Mean MVD /HPF (SD) \\
\hline Serous Carcinoma & 06 & 17.37 \\
\hline Mucinous Carcinoma & 05 & 16.33 \\
\hline Malignant Brenner Tumour & 01 & 11 \\
\hline Total & 12 & $16.53(2.36)$ \\
\hline
\end{tabular}

NOTE: Mean Micro vessel density per high power field of malignant ovarian surface epithelial tumours is $16.53 \pm 2.36$.

Table-7: Comparision of Micro Vessel Density of Ovarian Surface EpithelialTumours

\begin{tabular}{|l|l|}
\hline Type of Tumour & Mean MVD/HPF \\
\hline Benign & 4.48 \\
\hline Borderline & 7.5 \\
\hline Malignant & 16.5 \\
\hline
\end{tabular}

NOTE: Mean Micro Vessel Density of malignant Ovarian Surface Epithelial Tumours (16.5) is high compared to benign (4.48) and borderline tumours (7.5).

\section{DISCUSSION}

Ovarian cancer is a fatal gynecological malignancy. They are composed of many histological types, but majority are papillary serous carcinomas. The aim of the present study is to evaluate the microvessel density and maturity characteristics of proliferating blood vessels in benign and malignant surface epithelial tumours of ovary. The results thus obtained are analyzed and discussed in the light of other publications. During the 15 months study period, we received a total of 70 ovarian tumours, among which 34 are surface epithelial tumours. 4 cases are excluded from the study due to autolysis and the study is done on 30 cases. Incidence of ovarian surface epithelial tumours at our hospital is $48.57 \%$ which is most common among ovarian tumours similar to studies of Misra R. K et al., [11], Naseer. A. Shaikh et al., [12].

In this study, youngest age at diagnosis is 29 years and oldest is 65 years. Serous tumours are the most common (60\%) similar to seen in studies of Hollingsworth et al., Arjunan et al., [9], Sehgal et al., Emoto et al., Tejaswini et al., Misra R. K et al., [11].

Table-8: Incidence of benign and malignant ovarian surface epithelial tumours

\begin{tabular}{|l|l|l|l|l|}
\hline Study done by & Place of study & Benign & Malignant & Total \\
\hline Arjunan et al $^{14}$ & India & 32 & 18 & 50 \\
\hline Sehgal et al & India & 28 & 14 & 42 \\
\hline Emoto et al & Japan & 44 & 20 & 64 \\
\hline Present study & India & $18^{*}$ & 12 & 30 \\
\hline
\end{tabular}

$* 15$ cases of benign and 3 cases borderline tumours

Microvessel density is significantly higher in malignant Ovarian surface epithelial tumours compared to benign tumours. Blood vessels of malignant Ovarian surface epithelial tumours have decreased Smooth Muscle Actin expression as compared to benign tumours. Malignant tumours showed immature blood vessels and paucity of smooth muscle support. These results reveal that blood vessels in malignant tumours are thin walled, weak and haphazardly arranged, allowing easier spread of tumour cells.

Abulafia et al., [12] studied angiogenesis on ovarian tumours in 83 patients by calculating microvessel density using antibodies against vWF Study showed that angiogenic switch occurs between benign and borderline and malignant tumours and 
microvessel density might help to differentiate between benign and invasive tumours.

Wang et al., [13] did a study on characteristics of contrast enhanced ultrasound and its utility in assessing microvessel density in ovarian tumours which showed that microvessel density is higher in malignant tumours as compared to benign.

Sehgal et al., [14] conducted a study on 42 cases of ovarian surface epithelial tumours. Microvessel density was calculated using antibodies against CD 34. Result showed that malignant tumours showed higher microvessel density as compared to benign ovarian tumours and similar findings are obtained in our study.

Taylor proposed borderline category of surface epithelial tumours. They lack ovarian stromal invasion by definition. Morphologic criteria for diagnosing serous and mucinous borderline tumours vary according to histologic type. For serous type, stromal invasion by neoplastic epithelial cells is the main distinguishing feature between well-differentiated carcinoma from borderline tumours.
In our study, out of 30 cases- 3 cases of borderline surface epithelial tumours were diagnosed; 2 of serous and one of mucinous tumours. Due to very low number of cases, comparative study could not be done. However, the micro vessel density was found to be higher than benign tumours and lower than malignant tumours.

Studies have been done to assess and compare micro vessel density of benign and malignant ovarian surface epithelial neoplasms [15]. They concluded that average micro vessel density was significantly higher in malignant ovarian surface epithelial tumours and high micro vessel density was associated with transformation and acquisition of invasive phenotype of advanced epithelial ovarian cancers.

Arjunan et al., [9] conducted a study on CD 34 expression in surface epithelial tumours of ovary in 50 cases showed that mean micro vessel density was statistically significantly higher for malignant tumours when compared to benign.

Table-9: Supporting Studies of Micro Vessel Density

\begin{tabular}{|l|l|l|l|l|}
\hline Study & Sample size & Mean MVD / hpf - Beingn & Mean MVD / hpf - Malignant & P value \\
\hline Present study & 30 & $4.48 / 0.196 \mathrm{~mm}^{2}$ & $16.53 / 0.196 \mathrm{~mm}^{2}$ & $<0.001$ \\
\hline Sehgal et al., [14] & 42 & $16.53 / 0.375 \mathrm{~mm}^{2}$ & $34.5 / 0.375 \mathrm{~mm}^{2}$ & $<0.001$ \\
\hline Arjunan et al., $[9]$ & 50 & $14.88 / 0.375 \mathrm{~mm}^{2}$ & $26.09 / 0.375 \mathrm{~mm}^{2}$ & 0.002 \\
\hline
\end{tabular}

Recent studies have shown that measuring the micro vessel density for assessing the tumour angiogenesis as an important and independent prognostic factor for predicting tumour growth and metastasis. Assessment of micro vessel density by using immunohistochemical markers like CD34 were much more prominent and more easily counted than same vesssels stained with $\mathrm{H} \& \mathrm{E}$.

The prognostic value of micro vessel density was first assessed by Van Diest et al., [16] in advanced epithelial ovarian cancer. Micro vessel density is useful in assessing the degree of angiogenesis of tumour, hence micro vessel density is a powerful prognostic and predictive tool. Primary tumour with high micro vessel density is more likely to seed distant sites.

The routine use of $\mathrm{CD} 34$ expression as prognostic factor should be supported and analysed with further studies involving large sample size. No specific pathway or gene mutation has been reported to epithelial ovarian cancers. So, combination therapies and multi-targeted drugs may be appropriate.

Recent advances in treatment resulted in an improvement in 5 year survival in patients with epithelial ovarian cancer. The greatest success in epithelial ovarian cancer has come from targeting angiogenesis. Initial theoretical concerns arised that efforts to target vascular supply to tumours can lead to hypoxia or hypoperfusion causing resistance to radiotherapy and chemotherapy but, intratumoural blood vessels are different in structures function, permeability from their counterparts in normal tissues.

\section{CONCLUSION}

The study was done with aims of determining the incidence of Ovarian Surface Epithelial tumours at our hospital and to study the differences in angiogenesis and characteristics of tumour vessels between benign and malignant epithelial tumours using immunohistochemistry. Incidence of ovarian surface epithelial tumours at our hospital is $48.37 \%$. Serous tumours are most common tumours $(60 \%)$ followed by mucinous tumours $(40 \%)$. Malignant ovarian tumours showed higher production of immature blood vessels compared to benign tumours. Therefore Mean Micro Vessel Density is higher in malignant tumours as compare to benign tumours. In view of these results, new therapy should aim to alter ratio of immature to mature blood vessels and also Micro vessel density of malignant tumours for a successful outcome.

\section{REFERENCES}


1. Kumar, A. A. (2014). The Female Genital Tract: Robbins and Cotran Pathologic Basis of Disease, South Asia Edition 9. Reed Elsevier, 1023-8.

2. Kumar, R. V., Mukherjee, G., Umadevi, K., \& Hazarika, D. (1994). Sclerosing stromal tumour of the ovary. Indian journal of cancer, 31(2), 92-95.

3. Auersperg, N., Wong, A. S., Choi, K. C., Kang, S. K., \& Leung, P. C. (2001). Ovarian surface epithelium: biology, endocrinology, and pathology. Endocrine reviews, 22(2), 255-288.

4. Levanon, K., Crum, C., \& Drapkin, R. (2008). New insights into the pathogenesis of serous ovarian cancer and its clinical impact. Journal of Clinical Oncology, 26(32), 5284-93.

5. Bamberger, E. S., \& Perrett, C. W. (2002). Angiogenesis in epithelial ovarian cancer. Mod Pathol; 55:348-59.

6. Kristensen, G. B., \& Tropé, C. (1997). Epithelial ovarian carcinoma. The Lancet, 349(9045), 113117.

7. Krigman, H., Bentley, H., \& Robboy, S. J. (1994). Pathology of epithelian ovarian tumours. Clin Obstet Gynecol, 37:475-91.

8. Toi, M., Tominaga, T., \& Kashitani, J. (1993). Tumor angiogenesis is an independent prognostic indicator in primary breast carcinoma. International journal of cancer, 55(3), 371-374.

9. Arjunan, G. S. (2016). Thiriveni Balaji. Expression of p53 and CD34 in surface epithelial tumours of ovary. IOSR-JDMS, 15:1-13

10. Weidner, N., Carroll, P. R., Flax, J., Blumenfeld, W., \& Folkman, J. (1993). Tumor angiogenesis correlates with metastasis in invasive prostate carcinoma. The American journal of pathology, 143(2), 401-409.

11. Misra, R. K., Sharma, S. P., Gupta, U., Gaur, R., \& Mishra, S. D. (1991). Pattern of ovarian neoplasm in eastern UP. J Obstet Gynecol India, 30, 242-46.

12. Abulafia, O., Ruiz, J. E., Holcomb, K., Dimaio, T. M., Lee, Y. C., \& Sherer, D. M. (2000). Angiogenesis in early-invasive and lowmalignant-potential epithelial ovarian carcinoma. Obstetrics \& Gynecology, 95(4), 548552.

13. Wang, J., Lv, F., Fei, X., Cui, Q., Wang, L., Gao, X., .. \& Liu, A. (2011). Study on the characteristics of contrast-enhanced ultrasound and its utility in assessing the microvessel density in ovarian tumors or tumor-like lesions. International journal of biological sciences, 7(5), 600-606.

14. Sehgal, S., Goyal, P., Agarwal, R., Singh, S., Kumar, A., Gupta, R., ... \& Agrawal, D. (2013). Differences in the angiogenesis of benign and malignant ovarian surface epithelial tumors demonstrated by microvessel density and immunohistochemistry. $J \quad$ Interdiscipl Histopathol, 1(3), 145-152.

15. Weidner, N., Carroll, P. R., Flax, J., Blumenfeld, W., \& Folkman, J. (1993). Tumor angiogenesis correlates with metastasis in invasive prostate carcinoma. The American journal of pathology, 143(2), 401-409.

16. Shaikh, N. A., Hashmi, F., \& Samoo, R. P. (2007). Pattern of ovarian tumors: report of 15 years experience at Liaquat University Jamshoro. $J$ Liaquat Uni Med Health Sci, 6, 13-5. 